\title{
s-Sequence-Covering Mappings on Metric Spaces
}

\author{
Xiangeng Zhou*and Li Liu \\ Department of Mathematics, Ningde Normal University, Ningde 352100, China.
}

Received September 11, 2020; Accepted December 29, 2020;

Published online January 13, 2022.

\begin{abstract}
In this paper, we introduce and study s-sequence-covering mappings and 1 -s-sequence-covering mappings, obtain some characterizations of $s$-sequence-covering and compact images of metric spaces, and prove that every s-sequence-covering and compact mapping in first-countable spaces is a 1-s-sequence-covering mapping.
\end{abstract}

AMS subject classifications: 54A20, 54B15, 54C08, 54D55, 40A05, 26 A03

Key words: Statistical convergence, s-sequence-covering mappings, 1-s-sequence-covering mappings, compact mappings.

\section{Introduction}

Statistical convergence as a generalization of the usual notion of convergence was introduced by H. Fast [1] and H. Steinhaus [2]. There is not doubt that the study of statistical convergence and its various generalizations has become an active research area [3-8]. The original notion of statistical convergence was introduced for the real space $\mathbb{R}$. Generally speaking, this notion was extended in two directions. One is to discuss statistical convergence in more general spaces, for example, locally convex spaces [9], Banach spaces with the weak topologies $[6,10,11]$, and topological spaces $[5,7,8]$. The other is to consider generalized notions defined by various limit processes, for example, $A$-statistical convergence [12], lacunary statistical convergence [13], and $\lambda$-statistical convergence [14]. Perhaps, a most general notion of statistical convergence is ideal (or filter) convergence $[15,16]$. On the other hand, to find the internal characterizations of certain images of metric spaces is one of the central questions in general topology. F. Siwiec [17] introduced the concept of sequence-covering mappings. Thereafter, the research in this area has been well developed [18-22].

As we know, sequence-covering mappings, 1-sequence-covering mappings and sequentially quotient mappings are one of the most important tools to study certain images

*Corresponding author. Email addresses: 56667400@qq. com (Zhou X), 429378220@qq.com (Liu L) 
of metric spaces [19]. Recently, V. Renukadevi and B. Prakash defined two new sequencecovering mappings about statistical convergence as follows: Let $f: X \rightarrow Y$ be a mapping. The mapping $f$ is said to be a statistically sequence-covering mapping [23], if for a given sequence $\left\{y_{n}\right\}_{n \in \mathbb{N}}$ with $y_{n} \rightarrow y$ in $Y$, there exists a sequence $\left\{x_{n}\right\}_{n \in \mathbb{N}}$ which statistically converges to a point $x \in f^{-1}(y)$ and each $x_{n} \in f^{-1}\left(y_{n}\right)$; the mapping $f$ is said to be a statistically sequentially quotient mapping [24], if for a given sequence $y_{n} \rightarrow y$ in $Y$, there exists a sequence $x_{k} \rightarrow x \in f^{-1}(y)$ such that the sequence $\left\{f\left(x_{k}\right)\right\}_{k \in \mathbb{N}}$ is statistically dense in $\left\{y_{n}\right\}_{n \in \mathbb{N}}$. They discussed the relationship among sequence-covering mappings, statistically sequence-covering mappings and statistically sequentially quotient mappings, and studied their roles in the images of metric spaces.

Theorem 1.1 ([24]). Let $f: X \rightarrow Y$ be a statistically sequentially quotient and boundary-compact map. If the space $X$ is an open and compact-covering image of some metric space, then $f$ is a 1-sequence-covering map.

It is well known that we have the following result for the usual convergence.

Theorem 1.2 ([22]). The following are equivalent for a topological space X:

(1) $X$ is a sequence-covering and compact image of a metric space.

(2) $X$ is a 1-sequence-covering and compact image of a metric space.

(3) X has a point-star network consisting of point-finite cs-covers.

(4) X has a point-star network consisting of point-finite sn-covers.

We wonder if there are similar results for the case of statistical convergence? For this reason, this paper introduces and discusses s-sequence-covering mappings and 1$s$-sequence-covering mappings. It is expected that $s$-sequence-covering mappings and 1 -s-sequence-covering mappings shall also play an active role.

\section{Preliminaries}

In this paper, the set of all positive integers is denoted by $\mathbb{N}$, and the cardinality of the set $B$ is denoted by $|B|$. The definition of statistical convergence of sequences is based on the notion of asymptotic density of a set $A \subset \mathbb{N}$.

Definition 2.1 ([25]). Let $A \subset \mathbb{N}$ and $A(n)=\{k \in A: k \leq n\}$ for each $n \in \mathbb{N}$. Then $\underline{\delta}(A)=$ $\liminf _{n \rightarrow \infty}|A(n)| / n$ and $\bar{\delta}(A)=\limsup _{n \rightarrow \infty}|A(n)| / n$ are the lower and upper asymptotic density of the set $A$, respectively. If $\underline{\delta}(A)=\bar{\delta}(A)$, then $\delta(A)=\lim _{n \rightarrow \infty}|A(n)| / n$ is called the asymptotic density of $A$. A set $A \subset \mathbb{N}$ is said to be a statistically dense set if $\delta(A)=1$; a subsequence $\left\{x_{n_{k}}\right\}_{k \in \mathbb{N}}$ of a sequence $\left\{x_{n}\right\}_{n \in \mathbb{N}}$ is said to be statistically dense in $\left\{x_{n}\right\}_{n \in \mathbb{N}}$ if the set $\left\{n_{k}: k \in \mathbb{N}\right\}$ is statistically dense in $\mathbb{N}$.

Definition 2.2 ([5]). Let X be a topological space. 
(1) A sequence $\left\{x_{n}\right\}_{n \in \mathbb{N}}$ in $X$ is said to converge statistically (or shortly, s-converge) to a point $x \in X$, if $\delta\left(\left\{n \in \mathbb{N}: x_{n} \notin U\right\}\right)=0$ for each neighborhood $U$ of $x$ in $X$, which is denoted by $s-\lim _{n \rightarrow \infty} x_{n}=x$ or $x_{n} \stackrel{s}{\rightarrow} x$.

(2) A sequence $\left\{x_{n}\right\}_{n \in \mathbb{N}}$ in $X$ is said to $s^{*}$-converge to a point $x \in X$, if there is $A \subset \mathbb{N}$ with $\delta(A)=1$ and $\lim _{A \ni n \rightarrow \infty} x_{n}=x$, which is denoted by $x_{n} \stackrel{s^{*}}{\rightarrow} x$.

Remark 2.1. (1) If $A \subset \mathbb{N}$ and $\delta(A)$ exists, then $\delta(\mathbb{N} \backslash A)=1-\delta(A)$ and $0 \leq \delta(A) \leq 1$.

(2) The limit of a statistically convergent sequence is uniquely determined in Hausdorff spaces.

(3) If a sequence $\left\{x_{n}\right\}_{n \in \mathbb{N}}$ converges to $x$ in the usual sense, then it statistically converges to $x$; but the converse is not true in general.

(4) A sequence $\left\{x_{n}\right\}_{n \in \mathbb{N}}$ is statistically convergent if and only if each statistically dense subsequences of its is statistically convergent.

Lemma 2.1 ([5]). Let $X$ be a first-countable space and a sequence $\left\{x_{n}\right\}_{n \in \mathbb{N}} \subset X$. Then $x_{n} \stackrel{s}{\rightarrow} x$ if and only if $x_{n} \stackrel{s^{*}}{\rightarrow} x$.

Let $X$ be a topological space, $P \subset X$ and $x \in X$. $P$ is called a sequential neighborhood of $x$ in $X$ if whenever $\left\{x_{n}\right\}_{n \in \mathbb{N}}$ is a sequence converging to the point $x,\left\{x_{n}\right\}_{n \in \mathbb{N}}$ is eventually in $P$. A subset $F \subset X$ is called a sequentially closed set if $F$ is closed with respect to the usual convergence of sequences in $F$, i.e., for each sequence $\left\{x_{n}\right\}_{n \in \mathbb{N}} \subset F$ with $x_{n} \rightarrow x \in X$, $x \in F . \quad X$ is called a sequential space $[8,26]$ if each sequentially closed subset of $X$ is a closed set. A subset $U \subset X$ is called a sequentially open set if $X \backslash U$ is sequentially closed. Obviously, a subset $U \subset X$ is a sequentially open set if and only if for each sequence $\left\{x_{n}\right\}_{n \in \mathbb{N}}$ converging to a point $x \in U$, the sequence $\left\{x_{n}\right\}_{n \in \mathbb{N}}$ is eventually in $U$; a space $X$ is a sequential space if and only if each sequentially open subset of $X$ is open. Every first-countable space is a sequential space [24].

Definition 2.3 ([8]). Let X be a topological space.

(1) A subset $F \subset X$ is said to be an s-sequentially closed set if for each sequence $\left\{x_{n}\right\}_{n \in \mathbb{N}} \subset F$ with $x_{n} \stackrel{s}{\rightarrow} x \in X, x \in F$.

(2) A subset $U \subset X$ is said to be an s-sequentially open set if $X \backslash U$ is s-sequentially closed.

(3) $X$ is called an s-sequential space if each s-sequentially closed subset of $X$ is closed.

Obviously, every sequential space is an s-sequential space.

Definition 2.4. Let $X$ be a topological space and $P \subset X . P$ is called an s-sequential neighborhood of $x$, if for each sequence $\left\{x_{n}\right\}_{n \in \mathbb{N}}$ statistically converges to $x \in P, \delta\left(\left\{n \in \mathbb{N}: x_{n} \notin P\right\}\right)=0$.

Lemma 2.2. Let $X$ be a first-countable space and $P \subset X$. If $P$ is a sequential neighborhood of $x$ in $X$, then $P$ is an s-sequential neighborhood of $x$. 
Proof. Let $\left\{x_{n}\right\}_{n \in \mathbb{N}}$ be a sequence in $X$ with $x_{n} \stackrel{s}{\rightarrow} x$. By Lemma 2.1, there exists a set $M=$ $\left\{m_{k}: k \in \mathbb{N}\right\} \subset \mathbb{N}$ with $\delta(M)=1$ and $\lim _{k \rightarrow \infty} x_{m_{k}}=x$. Since $P$ is a sequential neighborhood of $x$ in $X$, there exists $k_{0} \in \mathbb{N}$ such that $\left\{x_{m_{k}}: k>k_{0}\right\} \cup\{x\} \subset P$, hence

$$
\left\{n \in \mathbb{N}: x_{n} \notin P\right\} \subset(\mathbb{N} \backslash M) \cup\left\{m_{1}, m_{2}, \cdots, m_{k_{0}}\right\} .
$$

Since $\delta(\mathbb{N} \backslash M)=1-\delta(M)=0$, it follows that $\delta\left((\mathbb{N} \backslash M) \cup\left\{m_{1}, m_{2}, \cdots, m_{k_{0}}\right\}\right)=0$. Thus $P$ is an s-sequential neighborhood of $x$.

Definition 2.5. Let $X, Y$ be topological spaces and $f: X \rightarrow Y$ be a mapping.

(1) $f$ is called a preserving s-convergence mapping provided for each sequence $\left\{x_{n}\right\}_{n \in \mathbb{N}}$ in $X$ with $x_{n} \stackrel{s}{\rightarrow} x$, the sequence $\left\{f\left(x_{n}\right)\right\}_{n \in \mathbb{N}} s$-converges to $f(x)$.

(2) $f$ is called an s-continuous mapping provided $U$ is an s-sequentially open set in $Y, f^{-1}(U)$ is an s-sequentially open set in $X$.

Lemma 2.3 ([7]). Every continuous mapping is a preserving s-convergence mapping. And every preserving s-convergence mapping is an s-continuous mapping.

Definition 2.6. Let X be a topological space and $\mathscr{P}$ be a cover of X.

(1) $\mathscr{P}$ is a cs-cover [27] of $X$ if for any convergent sequence $S$ in $X$, there exists $P \in \mathscr{P}$ such that $S$ is eventually in $P$.

(2) $\mathscr{P}$ is an sn-cover [22] of $X$ if each element of $\mathscr{P}$ is a sequential neighborhood of some point in $X$ and for each $x \in X$, there exists $P \in \mathscr{P}$ such that $P$ is a sequential neighborhood of $x$.

(3) $\mathscr{P}$ is an s-cs-cover of $X$ if whenever $\left\{x_{n}\right\}_{n \in \mathbb{N}}$ is a sequence in $X$ statistically converging to $x$, there exists $P \in \mathscr{P}$ such that $x \in P$ and $\delta\left(\left\{n \in \mathbb{N}: x_{n} \notin P\right\}\right)=0$.

(4) $\mathscr{P}$ is an s-sn-cover of $X$ if each element of $\mathscr{P}$ is an s-sequential neighborhood of some point of $X$ and for each $x \in X$, there exists $P \in \mathscr{P}$ such that $P$ is an s-sequential neighborhood of $x$.

Lemma 2.4. Let $n_{0} \in \mathbb{N}$ and $\left\{x_{m, n}\right\}_{n \in \mathbb{N}}$ be a sequence in $X$ with $x_{m, n} \stackrel{s}{\rightarrow} x_{0}$ for each $m \in$ $\left\{1, \cdots, n_{0}\right\}$. Put

$$
\left\{z_{k}\right\}_{k \in \mathbb{N}}=\left\{x_{1,1}, \cdots, x_{n_{0}, 1}, x_{1,2}, \cdots, x_{n_{0}, 2}, \cdots\right\},
$$

where $k=(n-1) n_{0}+m, m<n_{0}, n \in \mathbb{N}$. Then the sequence $z_{k} \stackrel{s}{\rightarrow} x_{0}$.

Proof. For each neighborhood $U$ of $x_{0}$ in $X$, it is not difficult to observe that

$$
\begin{aligned}
\left\{k \in \mathbb{N}: z_{k} \notin U\right\} & =\bigcup_{m=1}^{n_{0}}\left\{k=(n-1) n_{0}+m \in \mathbb{N}: z_{k} \notin U\right\} \\
& =\bigcup_{m=1}^{n_{0}}\left\{(n-1) n_{0}+m \in \mathbb{N}: x_{m, n} \notin U\right\} .
\end{aligned}
$$

For each $m \in\left\{1,2, \cdots, n_{0}\right\}$, since each $x_{m, n} \stackrel{s}{\rightarrow} x_{0}$, it follows that $\delta\left(\left\{n \in \mathbb{N}: x_{m, n} \notin U\right\}\right)=0$. Besides, for each $n, i \in \mathbb{N}$, it is easy to verify that $(n-1) n_{0}+m \leq n_{0} i \Leftrightarrow n \leq i$. Hence, for each $m \in\left\{1,2, \cdots, n_{0}\right\}$

$$
\left|\left\{n \in \mathbb{N}: x_{m, n} \notin U, n \leq i\right\}\right|=\left|\left\{(n-1) n_{0}+m \in \mathbb{N}: x_{m, n} \notin U,(n-1) n_{0}+m \leq n_{0} i\right\}\right| .
$$


Consequently,

$$
\begin{aligned}
& \delta\left(\left\{k \in \mathbb{N}: z_{k} \notin U\right\}\right) \\
\leq & \sum_{m=1}^{n_{0}} \delta\left(\left\{k=(n-1) n_{0}+m \in \mathbb{N}: z_{k} \notin U\right\}\right) \\
= & \sum_{m=1}^{n_{0}} \delta\left(\left\{(n-1) n_{0}+m \in \mathbb{N}: x_{m, n} \notin U\right\}\right) \\
= & \sum_{m=1}^{n_{0}} \lim _{i \rightarrow \infty} \frac{\left|\left\{(n-1) n_{0}+m \in \mathbb{N}: x_{m, n} \notin U,(n-1) n_{0}+m \leq n_{0} i\right\}\right|}{n_{0} i} \\
= & \sum_{m=1}^{n_{0}} \lim _{i \rightarrow \infty} \frac{\left|\left\{n \in \mathbb{N}: x_{m, n} \notin U, n \leq i\right\}\right|}{n_{0} i}=0 .
\end{aligned}
$$

Thus $z_{k} \stackrel{s}{\rightarrow} x_{0}$.

Throughout this paper, all spaces are assumed to be Hausdorff, and all mappings are surjection and continuous. The readers may refer to [28,29] for notation and terminology not explicitly given here.

\section{3 s-sequence-covering and compact images of metric spaces}

In this section, we mainly discuss $s$-sequence-covering and compact images of metric spaces. Let $X, Y$ be topological spaces and $f: X \rightarrow Y$ be a mapping. The mapping $f$ is said to be a sequence-covering mapping if whenever $\left\{y_{n}\right\}_{n \in \mathbb{N}}$ is a convergent sequence in $Y$, there is a convergent sequence $\left\{x_{n}\right\}_{n \in \mathbb{N}}$ in $X$ with each $x_{n} \in f^{-1}\left(y_{n}\right)$ [17]. The mapping $f$ is compact if $f^{-1}(y)$ is a compact subset in $X$ for each $y \in Y$.

Definition 3.1 ([7]). Let $X, Y$ be topological spaces and $f: X \rightarrow Y$ be a mapping. $f$ is said to be an s-sequence-covering mapping if whenever $\left\{y_{n}\right\}_{n \in \mathbb{N}}$ is a statistically convergent sequence in $Y$, there is a statistically convergent sequence $\left\{x_{n}\right\}_{n \in \mathbb{N}}$ in $X$ with each $x_{n} \in f^{-1}\left(y_{n}\right)$.

Two examples below show that sequence-covering mappings and s-sequence-covering mappings are independent.

Example 3.1. There exists a sequence-covering mapping which is not an s-sequencecovering mapping.

Proof. Let $S=\left\{x_{n}: n \in \mathbb{N}\right\}$ be a countable set. Take $x \notin S$ and put $X=S \cup\{x\}$. The topology on $X$ is defined as follows:

(1) each point $x_{n}$ is isolated;

(2) each open neighborhood $U$ of the point $x$ is of the form $\{x\} \cup M$, where $M \subset S$ and $\delta\left(\left\{n \in \mathbb{N}: x_{n} \in M\right\}\right)=1$. 
It was obtained that the space $X$ is a statistically sequential space but no sequence of $S$ converges to the point $x$ [8, Example 2.1] .

Now, let $Z$ be the set $X$ endowed with the discrete topology. Define a mapping $f$ : $Z \rightarrow X$ to be the identity mapping. Obviously, $f$ is a continuous mapping. Since there is no any non-trivial convergent sequence in $X, f$ is a sequence-covering mapping. But $f$ is not an s-sequence-covering mapping. In fact, the sequence $\left\{x_{n}\right\}_{n \in \mathbb{N}} \subset X$ s-converges to $x \in X$. But $\delta\left(\left\{n \in \mathbb{N}: x_{n} \neq x\right\}\right)=\delta(\mathbb{N})=1$. Consequently, $\left\{x_{n}\right\}_{n \in \mathbb{N}} \subset Z$ does not $s$-converge to $x \in Z$.

Example 3.2. There exists an s-sequence-covering mapping which is not a sequencecovering mapping.

Proof. Let $Y=\{0\} \cup\{1 / n: n \in \mathbb{N}\}$ be a subspace of $\mathbb{R}$ with the usual topology. Denote

$$
\left\{\overline{\left\{y_{k}: k \in \mathbb{N}\right\}}:\left\{y_{k}\right\}_{k \in \mathbb{N}} \subset Y \text { is a convergent sequence }\right\}=\left\{Y_{\alpha}: \alpha \in \Lambda\right\} .
$$

Obviously, $\left\{Y_{\alpha}: \alpha \in \Lambda\right\}$ is a cover of $Y$. For each $\alpha \in \Lambda$, the set $Y_{\alpha}$ is endowed with the following topology and denoted it by $X_{\alpha}$ : if $Y_{\alpha}$ is a finite set, then $X_{\alpha}$ is a discrete space; if $Y_{\alpha}$ is an infinite set, the topology on $X_{\alpha}$ is defined as Example 3.1 with $x_{0}=0$. Put the topological sum $X=\bigoplus_{\alpha \in \Lambda} X_{\alpha} \times\{\alpha\}$. Let $p: X \rightarrow Y$ be a natural mapping, that is, $p((y, \alpha))=y$ for each $(y, \alpha) \in X_{\alpha} \times\{\alpha\}$ and $\alpha \in \Lambda$.

Assume that $U$ is a neighborhood of 0 in $Y$. Then $Y \backslash U$ is a finite set, and further $\left(X_{\alpha} \times\{\alpha\}\right) \cap p^{-1}(Y \backslash U)$ is a finite set for each $\alpha \in \Lambda$. Thus $p^{-1}(Y \backslash U)$ is closed in $X$, and hence $p^{-1}(U)$ is open in $X$. Therefore $p$ is continuous.

In Example 3.1, it was mentioned that there is no any non-trivial convergent sequence in $X_{\alpha}$ for each $\alpha \in \Lambda$. Hence there is no any non-trivial convergent sequence in $X$. Consequently, $p$ is not a sequence-covering mapping.

Let $\left\{y_{k}\right\}_{k \in \mathbb{N}} \subset Y$ be an s-convergent sequence. Without loss of generality, we can assume that $y_{k} \stackrel{s}{\rightarrow} 0$. Since $Y$ is a first-countable space, by Lemma 2.1, there is $A \subset \mathbb{N}$ with $\delta(A)=1$ such that $\lim _{A \ni k \rightarrow \infty} y_{k}=0$. Hence, there exists $\alpha \in \Lambda$ such that $\left\{y_{k}: k \in A\right\} \cup$ $\{0\}=Y_{\alpha}$. Since the sequence $\left\{y_{k}\right\}_{k \in A}$ in $X_{\alpha}$ s-converges to 0 , the sequence $\left\{\left(y_{k}, \alpha\right)\right\}_{k \in A}$ $s$-converges to $(0, \alpha)$. For each $k \in \mathbb{N}$, put $x_{k} \in p^{-1}\left(y_{k}\right)$ satisfying $x_{k}=\left(y_{k}, \alpha\right) \in Y_{\alpha} \times\{\alpha\}$ as $k \in A$. And because $\delta(A)=1$, the sequence $\left\{x_{k}\right\}_{k \in \mathbb{N}}$ in $X$ s-converges to $(0, \alpha)$. Thus $p$ is an $s$-sequence-covering mapping.

Theorem 3.1. Let $f: X \rightarrow Y$ be an s-sequence-covering and compact mapping. Then for each $y \in Y$, there exists $x \in f^{-1}(y)$ such that if $U$ is an open neighborhood of $x$, then $f(U)$ is an s-sequential neighborhood of $y$.

Proof. Suppose not, that is, there exists $y \in Y$ such that for each $x \in f^{-1}(y)$, there exists an open neighborhood $U_{x}$ of $x$ such that $f\left(U_{x}\right)$ is not an s-sequential neighborhood of $y$. Since $f^{-1}(y) \subset \bigcup_{x \in f^{-1}(y)} U_{x}$ and $f$ is a compact mapping, there exists a finite subset $\left\{x_{m}\right.$ : $\left.m \leq n_{0}\right\}$ of $f^{-1}(y)$ such that $f^{-1}(y) \subset \bigcup_{m=1}^{n_{0}} U_{x_{m}}$. Since each $f\left(U_{x_{m}}\right)$ is not an s-sequential 
neighborhood of $y$, choose a sequence $\left\{y_{m, n}\right\}_{n \in \mathbb{N}}$ in $Y$ with $y_{m, n} \stackrel{s}{\rightarrow} y$ as $n \rightarrow \infty$, such that $\bar{\delta}\left(\left\{n \in \mathbb{N}: y_{m, n} \notin f\left(U_{x_{m}}\right)\right\}\right) \neq 0$ for each $m \in\left\{1,2, \cdots, n_{0}\right\}$ and $n \in \mathbb{N}$. Assume that

$$
1 \geq \bar{\delta}\left(\left\{n \in \mathbb{N}: y_{m, n} \notin f\left(U_{x_{m}}\right)\right\}\right)=\lambda_{m}>0
$$

for each $m \in\left\{1,2, \cdots, n_{0}\right\}$. Now define a sequence

$$
\left\{y_{1,1}, y_{2,1} \cdots, y_{n_{0}, 1}, y_{1,2}, \cdots, y_{n_{0}, 2}, \cdots\right\},
$$

and denote it by $\left\{y_{k}\right\}_{k \in \mathbb{N}}$, where $k=(n-1) n_{0}+m, m \leq n_{0}$ and $n \in \mathbb{N}$. By Lemma 2.4, it follows that $y_{k} \stackrel{s}{\rightarrow} y$. Since $f$ is an s-sequence-covering mapping, there exist $x \in f^{-1}(y)$ and $x_{k} \in f^{-1}\left(y_{k}\right)$ such that $x_{k} \stackrel{s}{\rightarrow} x$. Note that $x \in f^{-1}(y) \subset \bigcup_{m=1}^{n_{0}} U_{x_{m}}$, there exists $m_{0} \leq n_{0}$ such that $x \in U_{x_{m_{0}}}$. So that $\delta\left(\left\{k \in \mathbb{N}: x_{k} \notin U_{x_{m_{0}}}\right\}\right)=0$, and hence

$$
\delta\left(\left\{k \in \mathbb{N}: y_{k} \notin f\left(U_{x_{m_{0}}}\right)\right\}\right)=\delta\left(\left\{k \in \mathbb{N}: x_{k} \notin U_{x_{m_{0}}}\right\}\right)=0 .
$$

But this contradicts to

$$
\begin{aligned}
0 & <\frac{\lambda_{m_{0}}}{n_{0}}=\frac{1}{n_{0}} \bar{\delta}\left(\left\{n \in \mathbb{N}: y_{m_{0}, n} \notin f\left(U_{x_{m_{0}}}\right)\right\}\right) \\
& =\limsup _{n \rightarrow \infty} \frac{\left|\left\{n \in \mathbb{N}: y_{m_{0}, n} \notin f\left(U_{x_{m_{0}}}\right), n \leq i\right\}\right|}{n_{0} i} \\
& =\limsup _{n \rightarrow \infty} \frac{\left|\left\{(n-1) n_{0}+m_{0} \in \mathbb{N}: y_{m_{0}, n} \notin f\left(U_{x_{m_{0}}}\right),(n-1) n_{0}+m_{0} \leq n_{0} i\right\}\right|}{n_{0} i} \\
& =\bar{\delta}\left(\left\{k=(n-1) n_{0}+m_{0} \in \mathbb{N}: y_{k} \notin f\left(U_{x_{m_{0}}}\right)\right\}\right) \\
& \leq \bar{\delta}\left(\left\{k \in \mathbb{N}: y_{k} \notin f\left(U_{x_{m_{0}}}\right)\right\}\right) \\
& =\delta\left(\left\{k \in \mathbb{N}: y_{k} \notin f\left(U_{x_{m_{0}}}\right)\right\}\right) \\
& =0 .
\end{aligned}
$$

This completes the proof of the theorem.

Lemma 3.1. Let $\Gamma$ be an index set and $\left\{x_{\gamma, n}\right\}_{n \in \mathbb{N}}$ be a sequence in $X_{\gamma}$ for each $\gamma \in \Gamma$. Then the sequence $\left(x_{\gamma, n}\right)_{\gamma \in \Gamma} \stackrel{s}{\rightarrow}\left(x_{\gamma}\right)_{\gamma \in \Gamma} \in \prod_{\gamma \in \Gamma} X_{\gamma}$ if and only if each $x_{\gamma, n} \stackrel{s}{\rightarrow} x_{\gamma} \in X_{\gamma}(\gamma \in \Gamma)$.

Proof. Sufficiency. For any neighborhood $U$ of $\left(x_{\gamma}\right)_{\gamma \in \Gamma}$ in $\prod_{\gamma \in \Gamma} X_{\gamma}$, there exists a finite subset $\Gamma^{\prime} \subset \Gamma$ and an open set $U_{\gamma}$ in $X_{\gamma}\left(\gamma \in \Gamma^{\prime}\right)$ such that

$$
\left(x_{\gamma}\right)_{\gamma \in \Gamma} \in \prod_{\gamma \in \Gamma^{\prime}} U_{\gamma} \times \prod_{\gamma \in \Gamma \backslash \Gamma^{\prime}} X_{\gamma} \subset U .
$$

Since each $x_{\gamma, n} \stackrel{s}{\rightarrow} x_{\gamma}$, we have $\delta\left(\left\{n \in \mathbb{N}: x_{\gamma, n} \notin U_{\gamma}\right\}\right)=0$ for each $\gamma \in \Gamma^{\prime}$. Since

$$
\left\{n \in \mathbb{N}:\left(x_{\gamma, n}\right)_{\gamma \in \Gamma} \notin U\right\} \subset \bigcup_{\gamma \in \Gamma^{\prime}}\left\{n \in \mathbb{N}: x_{\gamma, n} \notin U_{\gamma}\right\},
$$


it follows that $\delta\left(\left\{n \in \mathbb{N}:\left(x_{\gamma, n}\right)_{\gamma \in \Gamma} \notin U\right\}\right)=0$. Thus $\left(x_{\gamma, n}\right)_{\gamma \in \Gamma} \stackrel{s}{\rightarrow}\left(x_{\gamma}\right)_{\gamma \in \Gamma \text {. }}$

Necessity. Let $p_{\gamma}: \prod_{\gamma \in \Gamma} X_{\gamma} \rightarrow X_{\gamma}$ be the projection mapping. Since $p_{\gamma}$ is continuous, by Lemma 2.3, it is a preserving s-convergence mapping. Hence, $x_{\gamma, n} \stackrel{s}{\rightarrow} x_{\gamma} \in X_{\gamma}$ for each $\gamma \in \Gamma$.

Let us recall the concept of point-star networks. Let $\mathscr{P}$ be a family of subsets of a set $X$ and $x \in X$. Put $(\mathscr{P})_{x}=\{P \in \mathscr{P}: x \in P\}$ and denote $\operatorname{st}(x, \mathscr{P})=\bigcup(\mathscr{P})_{x}$. A family $\mathscr{P}$ of subsets of a space $X$ is point-finite if $(\mathscr{P})_{x}$ is finite for each $x \in X ; \mathscr{P}$ is called a network at a point $x \in X$ [30] if $x \in \cap \mathscr{P}$ and for each neighborhood $U$ of $x$, there exists $P \in \mathscr{P}$ such that $P \subset U$. Let $\left\{\mathscr{P}_{n}\right\}$ be a sequence of covers of a space $X .\left\{\mathscr{P}_{n}\right\}$ is called a point-star network [22] of $X$ if $\left\langle\operatorname{st}\left(x, \mathscr{P}_{n}\right)\right\rangle$ is a network at $x$ in $X$ for each $x \in X^{+}$. Obviously, $\left\{\mathscr{P}_{n}\right\}$ is a point-star network of $X$ if and only if for each $x \in X$ and for given $P_{n} \in\left(\mathscr{P}_{n}\right)_{x},\left\langle P_{n}\right\rangle$ is a network at $x$ in $X[19]$.

Theorem 3.2. The following are equivalent for a space $X$ :

(1) $X$ is an s-sequence-covering and compact image of a metric space.

(2) X has a point-star network consisting of point-finite s-sn-covers.

(3) X has a point-star network consisting of point-finite s-cs-covers.

Proof. (1) $\Rightarrow(2)$. Suppose that $f: M \rightarrow X$ is an s-sequence-covering and compact mapping, where $M$ is a metric space. Then there exists a sequence $\left\{\mathscr{B}_{i}\right\}_{i \in \mathbb{N}}$ of locally finite open covers of $M$ such that for each compact subset $K$ of $M,\left\langle\operatorname{st}\left(K, \mathscr{B}_{i}\right)\right\rangle$ is a neighborhood base of $K$ in $M$. Put $\mathscr{P}_{i}=f\left(\mathscr{B}_{i}\right)$. As $f$ being a compact mapping, $\mathscr{P}_{i}$ is a point-finite cover of $X$. For each $x \in X$, let $V$ be an open neighborhood of $x$ in $X$. Since $f^{-1}(x)$ is a compact subset in $M$ and $f^{-1}(x) \subset f^{-1}(V)$, there exists $n \in \mathbb{N}$ such that $\operatorname{st}\left(f^{-1}(x), \mathscr{B}_{n}\right) \subset f^{-1}(V)$. Hence $\operatorname{st}\left(x, \mathscr{P}_{n}\right) \subset V$, thus $\left\langle\operatorname{st}\left(x, \mathscr{P}_{i}\right)\right\rangle$ is a network at $x$ in $X$. This implies that $\left\{\mathscr{P}_{n}\right\}$ is a point-star network of $X$.

For each $x \in X$, there exists $b \in f^{-1}(x)$ satisfying the condition in Theorem 3.1. Since each $\mathscr{B}_{i}$ is an open cover of $X$, there exists $B \in \mathscr{B}_{i}$ such that $b \in B$. Put $P=f(B)$. By Theorem 3.1, $P$ is an $s$-sequential neighborhood of $x$. Let

$$
\mathscr{P}_{i}^{\prime}=\left\{P \in \mathscr{P}_{i}: P \text { is an s-sequential neighborhood of some point in } X\right\} .
$$

Then $\mathscr{P}_{i}^{\prime}$ is a point- finite cover of $X$ and $\left\{\mathscr{P}_{i}^{\prime}\right\}$ is a point-star network consisting of pointfinite $s$-sn-covers of $X$.

$(2) \Rightarrow(3)$ is obvious by Definition 2.6.

$(3) \Rightarrow(1)$. Let $\left\{\mathscr{P}_{i}\right\}$ be a point-star network consisting of point-finite $s$-cs-covers of $X$. For each $i \in \mathbb{N}$, put $\mathscr{P}_{i}=\left\{\mathscr{P}_{\alpha}: \alpha \in \Lambda_{i}\right\}$ and each $\Lambda_{i}$ is endowed with the discrete topology. Put

$$
M=\left\{\alpha=\left(\alpha_{i}\right) \in \prod_{i \in \mathbb{N}} \Lambda_{i}:\left\langle P_{\alpha_{i}}\right\rangle \text { forms a network at some point } x_{\alpha} \text { in } X\right\},
$$

\footnotetext{
${ }^{+} \mathrm{A}$ set $\left\{x_{n}: n \in \mathbb{N}\right\}$ is simply expressed as $\left\langle x_{n}\right\rangle$ in this paper.
} 
then $M$, which is a subspace of the product space $\prod_{i \in \mathbb{N}} \Lambda_{i}$, is a metrizable space and the point $x_{\alpha}$ is unique for each $\alpha \in M$. Define a function $f: M \rightarrow X$ by $f(\alpha)=x_{\alpha}$. Then $f$ is a compact mapping [19, Lemma 3.3.2].

Next, we shall show that $f$ is an s-sequence-covering mapping. Let $\left\{x_{n}\right\}_{n \in \mathbb{N}} \subset X$ be a sequence satisfying $x_{n} \stackrel{s}{\rightarrow} x_{0} \in X$. Since $\left\{\mathscr{P}_{i}\right\}$ is a point-finite $s$-cs-covers of $X$, we can choose $\alpha_{j} \in \Lambda_{j}$ such that $x_{0} \in P_{\alpha_{j}}$ and $\delta\left(\left\{n \in \mathbb{N}: x_{n} \notin P_{\alpha_{j}}\right\}\right)=0$ for each $j \in \mathbb{N}$. Since $\left\{\mathscr{P}_{i}\right\}$ is a point-star network of $X$ and $P_{\alpha_{j}} \in\left(\mathscr{P}_{j}\right)_{x_{0}},\left\langle P_{\alpha_{j}}\right\rangle$ forms a network at $x_{0}$ in $X$. Let $\alpha=\left(\alpha_{j}\right) \in M$. Then $\alpha \in f^{-1}\left(x_{0}\right)$. Choose a sequence $\left\{\left(\alpha_{j, n}\right)\right\}_{n \in \mathbb{N}}$ in $M$ as follows: for each $j \in \mathbb{N}$,

$$
\alpha_{j, n}= \begin{cases}\alpha_{j}, & x_{n} \in P_{\alpha_{j}}, \\ \beta_{j}, & x_{n} \in P_{\beta_{j}}, \text { for some } \beta_{j} \in \Lambda_{j} .\end{cases}
$$

Then $\alpha_{j, n} \stackrel{s}{\rightarrow} \alpha_{j}$ for each $j \in \mathbb{N}$, because $\delta\left(\left\{n \in \mathbb{N}: \alpha_{j, n} \notin V_{j}\right\}\right) \leq \delta\left(\left\{n \in \mathbb{N}: \alpha_{j, n} \neq \alpha_{j}\right\}\right)=$ $\delta\left(\left\{n \in \mathbb{N}: x_{n} \notin P_{\alpha_{j}}\right\}\right)=0$, if $V_{j}$ is a neighborhood of $\alpha_{j}$ in $\Lambda_{j}$. It follows from Lemma 3.1 that $\left(\alpha_{j, n}\right) \stackrel{s}{\rightarrow}\left(\alpha_{j}\right)$ in $M$. By the choice of $\left(\alpha_{j, n}\right)$, it is easy to see that $P_{\alpha_{j, n}} \in\left(\mathscr{P}_{j}\right)_{x_{n}}$, hence $\left\langle P_{\alpha_{j, n}}\right\rangle$ forms a network at $x_{n}$ in $X$, thus $\left(\alpha_{j, n}\right) \in f^{-1}\left(x_{n}\right)$ for each $n \in \mathbb{N}$. Therefore, $f$ is an $s$-sequence-covering mapping.

Definition 3.2. Let $X, Y$ be topological spaces and $f: X \rightarrow Y$ be a mapping. $f$ is said to be an s-quotient mapping provided $f^{-1}(U)$ is s-open in $X$, then $U$ is s-open in $Y$.

The following two theorems can be seen in [7].

Theorem 3.3. Each s-sequence-covering mapping is an s-quotient mapping.

Theorem 3.4. Let $X, Y$ be topological spaces and $f: X \rightarrow Y$ be a mapping.

(1) If $X$ is an s-sequential space and $f$ is a quotient mapping, then $Y$ is an s-sequential space and $f$ is an s-quotient mapping.

(2) If $Y$ is an s-sequential space and $f$ is an s-quotient mapping, then $f$ is a quotient mapping.

By Theorems 3.2, 3.3 and 3.4, we have the following corollary.

Corollary 3.1. The following are equivalent for a topological space X:

(1) $X$ is an s-sequence-covering, quotient and compact image of a metric space.

(2) $X$ is a sequential space and has a point-star network consisting of point-finite s-sn-covers.

(3) $X$ is a sequential space and has a point-star network consisting of point-finite s-cs-covers.

\section{1-s-sequence-covering mappings on first-countable spaces}

The work of this section is a continuation of the previous section. In this section, we obtain that $s$-sequence-covering and compact mappings in first-countable spaces are 1$s$-sequence-covering mappings. Recall the notion of 1-sequence-covering mappings in topological spaces. A mapping $f: X \rightarrow Y$ is a 1-sequence-covering mapping if for each $y \in Y$, there is $x \in f^{-1}(y)$ such that whenever $\left\{y_{n}\right\}$ is a sequence converging to $y$ in $Y$ there is a sequence $\left\{x_{n}\right\}$ converging to $x$ in $X$ with each $x_{n} \in f^{-1}\left(y_{n}\right)$ [18]. 
Definition 4.1. A mapping $f: X \rightarrow Y$ is called a 1-s-sequence-covering mapping if for each $y \in Y$, there is $x \in f^{-1}(y)$ such that whenever $\left\{y_{n}\right\}$ is a sequence statistically converging to $y$ in $Y$ there is a sequence $\left\{x_{n}\right\}$ statistically converging to $x$ in $X$ with each $x_{n} \in f^{-1}\left(y_{n}\right)$.

Obviously, if $f$ is a 1-s-sequence-covering mapping, then $f$ is an $s$-sequence-covering mapping. Two examples below show that 1 -sequence-covering mappings and 1-s-sequencecovering mappings are independent.

Example 4.1. There exists a 1-sequence-covering mapping in a first-countable space which is not an s-sequence-covering mapping.

Proof. Let $f: Z \rightarrow X$ be the mapping in Example 3.2. Then $Z$ is a first-countable space. Example 3.2 showed the mapping $f$ is not an $s$-sequence-covering mapping. Since there is no any non-trivial convergent sequence in $X, f$ is a 1-sequence-covering mapping.

Example 4.2. There exists a 1-s-sequence-covering mapping which is not a sequencecovering mapping.

Proof. Let $X=\{x\} \cup\left\{x_{n}: n \in \mathbb{N}\right\}$ be the topological space defined in Example 3.2. $Y=$ $\{0\} \cup\{1 / n: n \in \mathbb{N}\}$ be a subspace of $\mathbb{R}$ with the usual topology. Define a mapping $f: X \rightarrow Y$ by $f(x)=0$ and $f\left(x_{n}\right)=1 / n$ for each $n \in \mathbb{N}$. Since there is no any non-trivial convergent sequence in $X, f$ is not a sequence-covering mapping.

For each $y \in Y$, without loss of generality, we can assume that $y=0$. Take $x \in X$. If $\left\{y_{n}\right\}_{n \in \mathbb{N}} \subset Y$ is a sequence statistically converging to 0 . Since $Y$ is a first-countable space, by Lemma 2.1, there exists $A \subset \mathbb{N}$ with $\delta(A)=1$ and $\lim _{A \ni n \rightarrow \infty} y_{n}=0$. Assume that $\left\{y_{n}\right\}_{n \in \mathbb{N}}=\left\{1 / n_{i}\right\}_{i \in \mathbb{N}}$. Then $f\left(x_{n_{i}}\right)=1 / n_{i}$ for each $i \in \mathbb{N}$. Since $\delta(A)=1,\left\{x_{n_{i}}\right\}_{i \in \mathbb{N}}$ contains a statistically dense subsequence. It follows from Remark 2.1(4) that $x_{n_{i}} \stackrel{s}{\rightarrow} x$. Thus $f$ is a 1 -s-sequence-covering mapping.

Lemma 4.1. Let $f: X \rightarrow Y$ be a 1-s-sequence-covering mapping. Then for each $y \in Y$, there exists $x \in f^{-1}(y)$ such that whenever $U$ is an open neighborhood of $x$ in $X, f(U)$ is an s-sequential neighborhood of $y$ in $Y$.

Proof. Let $y \in Y$. Since $f$ is a 1-s-sequence-covering, there is $x \in f^{-1}(y)$ satisfying the condition in Definition 4.1. Let $U$ be an open neighborhood of $x$ in $X$ and $\left\{y_{n}\right\}$ be a sequence statistically converging to $y$ in $Y$. There is a sequence $\left\{x_{n}\right\}$ statistically converging to $x$ in $X$ with each $x_{n} \in f^{-1}\left(y_{n}\right)$. Hence $\delta\left(\left\{n \in \mathbb{N}: x_{n} \notin U\right\}\right)=0$, and further $\delta\left(\left\{n \in \mathbb{N}: y_{n} \notin f(U)\right\}\right) \leq \delta\left(\left\{n \in \mathbb{N}: x_{n} \notin U\right\}\right)=0$. Therefore, $f(U)$ is an s-sequential neighborhood of $y$ in $Y$.

Lemma 4.2. Let $f: X \rightarrow Y$ be a mapping and $\left\{B_{n}\right\}_{n \in \mathbb{N}}$ be a decreasing network at some point $x$ in $X$. If $\left\{y_{i}\right\}_{i \in \mathbb{N}}$ is a sequence in $Y$ statistically converging to $f(x)$ with each $\delta(\{i \in \mathbb{N}$ : $\left.\left.y_{i} \notin f\left(B_{n}\right)\right\}\right)=0$, then there is a sequence $\left\{x_{i}\right\}_{i \in \mathbb{N}}$ statistically converging to $x$ in $X$ with each $x_{i} \in f^{-1}\left(y_{i}\right)$. 
Proof. Let $\left\{y_{i}\right\}_{i \in \mathbb{N}}$ be a sequence in $Y$ statistically converging to $f(x)$ and $\delta\left(\left\{i \in \mathbb{N}: y_{i} \notin\right.\right.$ $\left.\left.f\left(B_{n}\right)\right\}\right)=0$ for each $n \in \mathbb{N}$. Note that each $f\left(B_{n}\right) \supset f\left(B_{n+1}\right)$. For each $i \in \mathbb{N}$, we can pick

$$
x_{i} \in \begin{cases}f^{-1}\left(y_{i}\right), & y_{i} \notin f\left(B_{1}\right), \\ f^{-1}\left(y_{i}\right) \cap B_{n}, & y_{i} \in f\left(B_{n}\right) \backslash f\left(B_{n+1}\right), n \in \mathbb{N} .\end{cases}
$$

For each $n \in \mathbb{N}$, if $x_{i} \notin B_{n}$, then $y_{i} \notin f\left(B_{n}\right)$. Otherwise, if $y_{i} \in f\left(B_{n}\right)$, then there exists $k \geq n$ such that $y_{i} \in f\left(B_{k}\right) \backslash f\left(B_{k+1}\right)$, thus $x_{i} \in B_{k} \subset B_{n}$, a contradiction; if $y_{i} \notin f\left(B_{n}\right)$, then $f^{-1}\left(y_{i}\right) \cap B_{n}=\varnothing$, hence $x_{i} \notin B_{n}$. Hence, by the choosing of $x_{i}$, it follows that $x_{i} \notin B_{n}$ if and only if $y_{i} \notin f\left(B_{n}\right)$ for each $n \in \mathbb{N}$. Thus, $x_{i} \stackrel{s}{\rightarrow} x$. In fact, for each open neighborhood $U$ of $x$, there exists $n_{0} \in \mathbb{N}$ such that $x \in B_{n_{0}} \subset U$. Therefore, $\left\{i \in \mathbb{N}: x_{i} \notin U\right\} \subset\left\{i \in \mathbb{N}: x_{i} \notin B_{n_{0}}\right\}=\left\{i \in \mathbb{N}: y_{i} \notin f\left(B_{n_{0}}\right)\right\}$, hence $\delta\left(\left\{i \in \mathbb{N}: x_{i} \notin U\right\}\right)=0$, and further $\left\{x_{i}\right\}_{i \in \mathbb{N}}$ statistically converges to $x$ in $X$ with each $x_{i} \in f^{-1}\left(y_{i}\right)$.

Corollary 4.1. Let $f: X \rightarrow Y$ be a mapping and $\left\{B_{n}\right\}_{n \in \mathbb{N}}$ be a decreasing network at some point $x$ in $X$. If $\left\{y_{i}\right\}_{i \in \mathbb{N}}$ is a sequence in $Y$ statistically converging to $f(x)$ and $f\left(B_{n}\right)$ is an s-sequential neighborhood of $f(x)$ in $Y$ for each $n \in \mathbb{N}$. Then there is a sequence $\left\{x_{i}\right\}_{i \in \mathbb{N}}$ statistically converging to $x$ in $X$ with each $x_{i} \in f^{-1}\left(y_{i}\right)$.

Theorem 4.1. Let $f: X \rightarrow Y$ be an s-sequence-cover and compact mapping. If $X$ is a first-countable space, then $f$ is a 1-s-sequence-cover mapping.

Proof. By Theorem 3.1, it follows that for each $y \in Y$, there exists $x \in f^{-1}(y)$ such that if $U$ is an open neighborhood of $x, f(U)$ is an s-sequential neighborhood of $y$. Let $\left\langle B_{n}\right\rangle$ be a decreasing open neighborhood base at $x$ in $X$. Then $f\left(B_{n}\right)$ is an s-sequential neighborhood of $f(x)$ in $Y$ for each $n \in \mathbb{N}$. By Corollary 4.1, if $\left\{y_{i}\right\}_{i \in \mathbb{N}}$ is a sequence in $Y$ statistically converging to $y$, there is a sequence $\left\{x_{i}\right\}_{i \in \mathbb{N}}$ statistically converging to $x$ in $X$ with each $x_{i} \in f^{-1}\left(y_{i}\right)$. Therefore, $f$ is a 1-s-sequence-cover mapping.

By Theorems 3.2 and 4.1, it is easy to obtain the following corollary.

Corollary 4.2. The following are equivalent for a topological space X:

(1) $X$ is a 1-s-sequence-covering and compact image of a metric space.

(2) $X$ is an s-sequence-covering and compact image of a metric space.

(3) X has a point-star network consisting of point-finite s-sn-covers.

(4) X has a point-star network consisting of point-finite s-cs-covers.

\section{Acknowledgments}

Our gratitude goes to Professor Shou Lin for his friendly encouragement and inspiring suggestions. This research is supported by the Natural Science Foundation of Fujian Province (Grant No. 2020J01428) and Ningde Normal University (Grant No. 2019ZDK11). 


\section{References}

[1] Fast H. Sur la convergence statistique. Colloq. Math., 1951, 2(3-4): 241-244.

[2] Steinhaus H. Sur la convergence ordinaire et la convergence asymptotique. Colloq. Math., 1951, 2 (1): 73-74.

[3] Cheng L X, Lin G C, Lan Y Y, et al. Measure theory of statistical convergence. Sci. China Ser. A, 2008, 51 (12): 2285-2303.

[4] Zhou X G, ZhanG M. More about the kernel convergence and the ideal convergence. Acta Math. Sinica, English Series, 2013, 29(12): 2367-2372.

[5] Maio Di G, Kocinac L D R. Statistical convergence in topology. Topol. Appl., 2008, 156(1): 28-45.

[6] Connor J, Ganivhev M, Kadets V. A characterization of Banach spaces with separable duals via weak statistical convergence. J Math. Anal. Appl., 2000, 44(1): 251-261.

[7] Liu L, Tang Z B, Lin S. Statistically sequential spaces and statistically sequentially quotient mappings(in Chinese). Applied Math. J. Chin. Univ., 2015, 30(4): 485-493.

[8] Tang Z B, Lin F C. Statistical versions of sequential and Fréchet-Urysohn spaces. Adv. Math. (China), 2015, 44(6): 945-954.

[9] Maddoox I J. Statistical convergence in a locally convex space. Math. Proc. Cambridge Philos. Soc., 1988, 104(1): 141-145.

[10] Kadets V. Weak cluster points of a sequence and covering by cylinder. Mat. Fiz. Anal. Geom., 2004, 11 (2): 161-168.

[11] Kadets V, Leonov A, Orhan C. Weak statistical convergence and weak filter convergence for unbounded sequences. J Math. Anal. Appl., 2010, 371(2): 414-424.

[12] Connor J On strong matrix summability with respect to a modulus and statistical convergence. Canad. Math. Bull., 1989, 32(2): 194-198.

[13] Fridy J, Orhan C. Lacunary statistical convergence. Pacific J. Math., 1993, 160(1): 43-51.

[14] Mursaleen M. $\lambda$-statistical convergence. Math. Slovaca, 2000, 50(1): 111-115.

[15] Kostyrko P, Salát T, WLICZYNSHI W. I-convergence. Real Anal. Exch., 2000/2001, 26(2): 669-686.

[16] Nurray F, Ruckle W H. Generalized statistical convergence and convergence free spaces. J. Math. Anal. Appl., 2000, 245(2): 513-527.

[17] Siwiec F. Sequence-covering and countably bi-quotient maps. General Topology Appl., 1971, 1(2): 143-154.

[18] Lin S. On sequence-covering s-maps. Adv. Math (China), 1996, 25(6): 548-551.

[19] Lin S. Point-Countable Covers and Sequence-Covering Mappings. Science Press, Beijing, 2015 (in Chinese, the second edition).

[20] Lin F C, Lin S. Sequence-covering maps on generalized metric spaces. Houston J. Math., 2014, 40(3): 927-943.

[21] Lin S, Yan P F. Sequence-covering maps of metric spaces. Topology Appl., 2001, 109: 301-314.

[22] Lin S, Yan P F. On sequence-covering and compact maps (in Chinese). Acta Math. Sinica., 2001, 44(1): 175-182.

[23] Renukadevi V, Prakash B. On statistically sequentially covering maps. Filomat, 2017, 31(6): 1681-1686.

[24] Renukadevi V, Prakash B. On statistically sequentially quotient maps. Korean J. Math., 2017, 25(1): 61-70.

[25] Niven I, Zuckerman H S, Montgomery H L. An Introduction to the Theory of Numbers (5th Ed.,). John Wiley and Sons, New York, 1991. 
[26] Franklin S P. Spaces in which sequences suffice. Fund. Math., 1965, 57: 107-115.

[27] Yan P F. On strong sequence-covering compact mappings. Northeastern Math. J., 1998, 14(3): 341-344.

[28] Engelking R. General Topology (revised and completed edition). Heldermann Verlag, Berlin, 1989.

[29] Lin S, Yun Z Q. Generalized Metric Spaces and Mapping. Atlantis Studies in Mathematics 6, Atlantis Press, Paris, 2016.

[30] Michael E. $\aleph_{0}$-spaces. J. Math. Mech., 1966, 15: 983-1002. 